\title{
Terapia antiangiogênica associada à fotocoagulação a laser no tratamento de membrana neovascular sub-retiniana: relato de caso
}

\author{
Anti-angiogenic therapy associated with laser photocoagulation in the treatment \\ of subretinal neovascular membrane: case report
}

José Alexandre Bastos Pereira ${ }^{1}$, Isabela Soutello Camarota²

\section{RESUMO}

O objetivo deste relato é descrever um caso de degeneração macular relacionada à idade com presença de membrana neovascular sub-retiniana (MNVSR) justafoveal, tratada inicialmente com bevacizumab (Avastin ${ }^{\circledR}$ ) intravítrea e posteriormente com terapia de fotocoagulação focal (TFC) com laser. A eficácia do tratamento inicial e complementar foi avaliada pela medida da acuidade visual e por exames complementares (angiografia fluoresceínica e tomografia de coerência óptica). Após três injeções intravítreas de bevacizumab ocorreu a redução excêntrica da MNVSR, alterando sua classificação para extrafoveal, o que permitiu o tratamento subsequente com TFC.

Descritores: Degeneração macular; Neovascularização coroidal; Inibidores de angiogênese/uso terapêutico; Angiofluoresceinografia; Tomografia de coerência óptica; Relato de caso

\begin{abstract}
The aim of this report is to describe a case of age-related macular degeneration with juxtafoveal subretinal neovascular membrane, initially treated with intravitreal bevacizumab (Avastin ${ }^{\circledR}$ ) and afterwards with focal photocoagulation laser therapy. The efficacy of the initial and complementary treatment was evaluated through the visual acuity measurement and complementary exams (fluorescein angiography and optical coherence tomography). After three bevacizumab intravitrealinjections, it has occurred theeccentric reduction of the neovascular subretinal membrane, changing its classification to extrafoveal, what allowed the subsequent treatment with focal photocoagulation laser therapy.
\end{abstract}

Keywords: Macular degeneration; Choroidal neovascularization; Angiogenesis inhibitors/ therapeutic use; Angiofluoresceinography; Tomography, optical coherence; Case report

\section{INTRODUÇÃO}

A degeneração macular relacionada à idade (DMRI) é uma doença degenerativa da retina que leva à perda da visão central, sendo a principal causa de cegueira irreversível nos pacientes com mais de 65 anos nos países desenvolvidos ${ }^{(1)}$. Essa patologia é dividida em forma não exsudativa e exsudativa. A forma seca ou não-exsudativa é caracterizada pela presença de lesão atrófica de contornos geográficos na região macular e ausência de membrana neovascular coroidal (MNVC). Ocorre na grande maioria dos casos e apresenta uma evolução lenta. A forma exsudativa é caracterizada pela formação de membrana neovascular sub-retiniana. Esta forma, embora tenha prevalência menor que a forma seca, é responsável por cerca de $80 \%$ dos casos de cegueira legal devido à degeneração macular relacionada à idade(2). O fator de crescimento endotelial (VEGF) constitui o principal estímulo angiogênico para a neovascularização na DMRI ${ }^{(3)}$. O bevacizumab (Avastin ${ }^{\circledR}$, Genentech Inc) um anticorpo monoclonal humanizado que se liga ao VEGF, atualmente vem sendo usado na inibição do VEGF apresentando bons resultados anatômicos e clínicos, inclusive com possibilidade de ganho de linhas de visão, mesmo em pacientes com membrana neovascular sub-retiniana (MNVSR) ocultas ${ }^{(4-7)}$. A terapia de fotocoagulação (TFC) focal com laser para tratamento da DMRI foi testada antes da década de 2000. Apesar dos resultados positivos iniciais para o tratamento de neovascularização justafoveal clássica, houve tendência à recorrência da lesão após o tratamento e prognóstico visual final pobre ${ }^{(8)}$.
Este estudo objetiva demonstrar a eficácia do tratamento com bevacizumab (Avastin ${ }^{\circledR}$ ) intravítrea associado à terapia de fotocoagulação (TFC) focal num caso de MNSR secundária a DMRI.

\section{RELATO DE CASO}

R.B.S., 70 anos de idade, sexo masculino, branco, natural e procedente do Rio de Janeiro, referindo diminuição da acuidade visual e metamorfopsia no olho esquerdo (OE) há cerca de 2 meses, sem outros sintomas associados. Portador de hipertensão arterial sistêmica, controlada clinicamente com uso de anti-hipertensivo oral, negava uso de outras medicações. Negava doenças oftalmológicas prévias. Ao exame oftalmológico apresentava acuidade visual (AV) com melhor correção óptica de 0,8 no olho direito (OD) e 0,1 no OE. A biomicroscopia de segmento anterior evidenciava catarata nuclear incipiente em ambos os olhos. A pressão intraocular era de 13 e $15 \mathrm{mmHg}$ às 13 horas. Ao exame fundoscópico do OD, foi evidenciado disco óptico de contornos nítidos e coloração normal, escavação fisiológica, presença de drusas duras no polo posterior, sem demais alterações. OE apresentava disco óptico de contornos nítidos e coloração normal, escavação fisiológica, área elevada de limites bem definidos no polo posterior, associada à presença de hemorragias e exsudatos sub-retinianos na borda, além de drusas no polo posterior. A angiografia fluoresceínica revelou em ambos os olhos presença de drusas hiperfluorescentes nas fases circulatórias, congruentes na região macular. OD apresentava área de dege- 
neração epitelial, proporcionando hiperfluorescência por defeito "em janela" nas fases circulatórias, em quadro de DMRI. No OE observou-se descolamento seroso em região macular e hemorragias subretinianas hipofluorescentes em todas as fases do exame, provenientes de MNSR do tipo clássica, justafoveal, gerando hiperfluorescência progressiva em intensidade e extensão. Procedeu-se ao estudo com a tomografia de coerência óptica (OCT) onde foi encontrada em OE presença de MNSR com área bem delimitada de elevação do epitélio pigmentário da retina nasal à fóvea e fluido subsensorial ao redor da lesão (Figura 1A). O OD apresentava perfil macular de contorno e espessura normais. O paciente foi orientado sobre riscos e benefícios da terapia com bevacizumab, e optou-se pelo tratamento. Foi aplicada injeção intravítrea de 1,25 mg de bevacizumab (Avastin ${ }^{\circledR}$ ) no OE sem nenhuma intercorrência. Após um mês do procedimento, o paciente apresentava acuidade visual de 0,5 e os exames complementares (angiografia fluoresceínica e OCT) demonstravam presença de MNSR ainda em atividade. Foram realizadas mais duas aplicações da terapia antiangiogênica, no período de dois meses, devido à persistência do edema macular e atividade da membrana neovascular. Quatro dias após a $3^{\underline{a}}$ injeção, a membrana neovascular sub-retiniana apresentava-se como um remanescente extrafoveal (Figura 1B). A OCT revelava presença de elevação do epitélio pigmentário da retina nasal à fóvea, e fluido subsensorial. AV era de 0,6. Foi então realizada terapia de fotocoagulação focal com laser na membrana extrafoveal. Os exames evidenciavam MNSR sem sinais de atividade, e redução significativa do edema macular, comparado aos exames anteriores (Figura 2). AV foi a 0,8 Dois anos após a última intervenção o paciente segue sem queixas e mantêm a AV de 0,8 no olho esquerdo.

\section{DISCUSSÃO}

O desenvolvimento de uma terapêutica efetiva e duradoura para o tratamento de DMRI persiste sendo um grande desafio para os oftalmologistas na atualidade. O tratamento com injeções intravítreas de agentes antiangiogênicos trouxe novas perspectivas para o manejo da forma exsudativa da doença. Embora estudos tenham demonstrado o ganho de mais de uma a duas linhas de AV e preservação visual em comparação a valores basais pré-tratamento(9), seu sucesso exige diversas aplicações; o que aumenta o risco global do tratamento, eleva seu custo e pode levar à perda da sua eficácia a longo prazo ${ }^{(10)}$. A TFC aplicada no presente caso agiu como importante fator adjuvante no controle da doença. Por ser uma doença de grande complexidade, a DMRI requer a abordagem individualizada

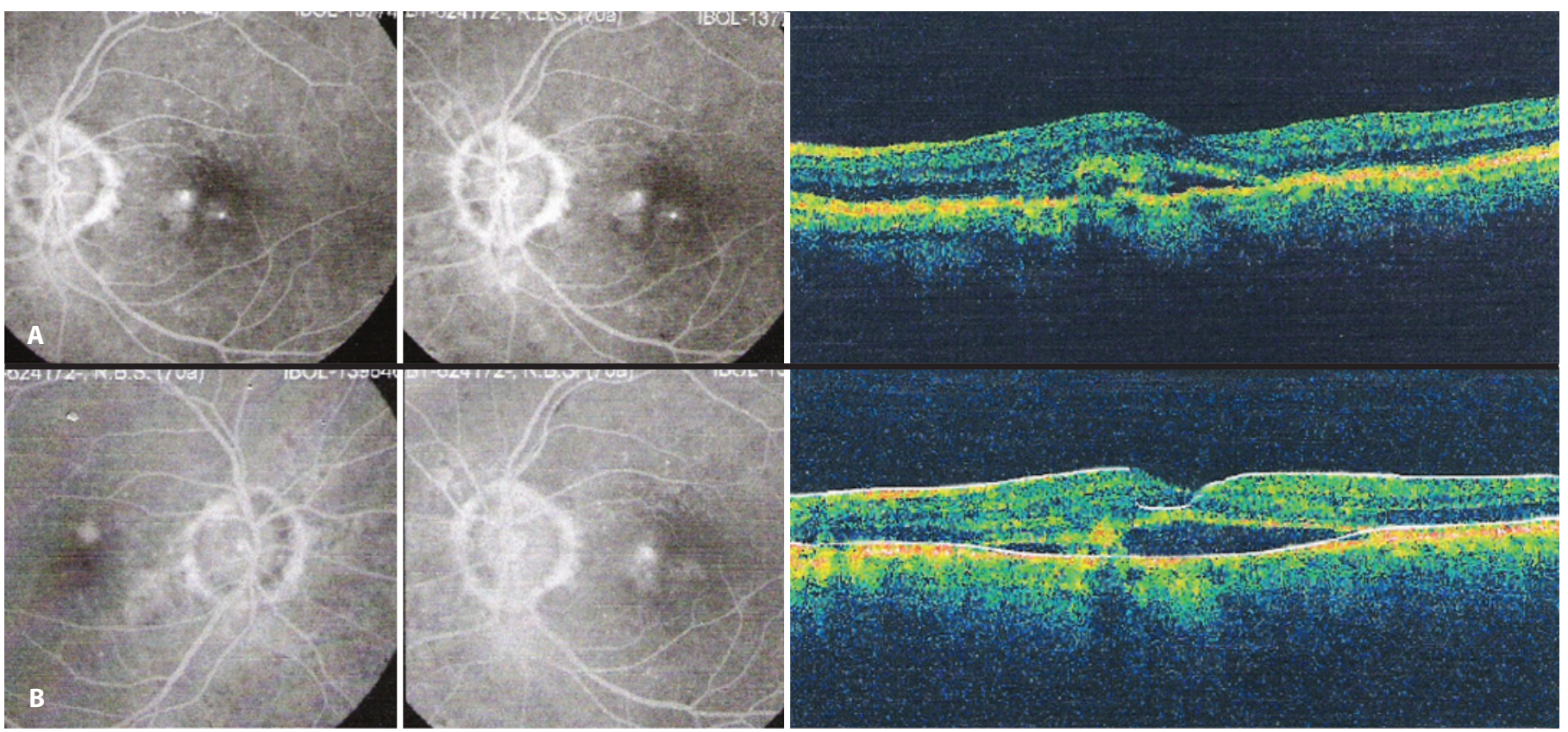

Figura 1. A) 24/11/06 - Apresentação inicial (AV 0,1 em OE); B) 15/05/07 - Após $3^{\underline{a}}$ injeção de bevacizumab (AV 0,6 em OE).
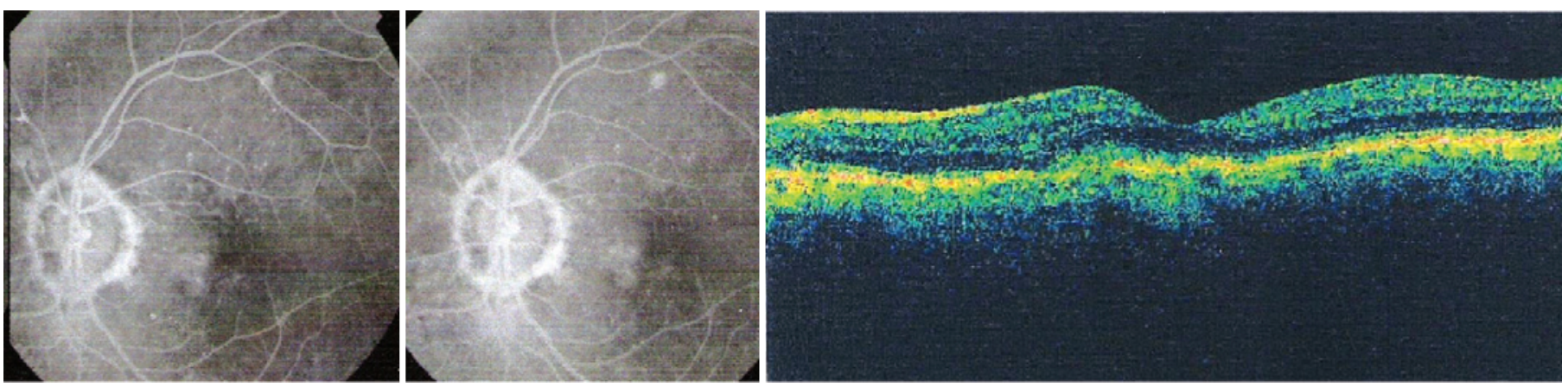

Figura 2. 13/08/07 - Após fotocoagulação focal (AV 0,8 em OE). 
de cada paciente para que seja obtido o melhor resultado clínico possível.

\section{REFERÉNCIAS}

1. Clemons TE, Milton RC, Klein R, Seddon JM, Ferris FL $3^{\text {rd }}$; Age-Related Eye Disease Study Research Group. Risk factors for the incidence of Advanced Age-Related Macular Degeneration in the Age-Related Eye Disease Study (AREDS) AREDS report n․19. Ophthalmology. 2005;112(4):533-9.

2. Guyer DR, Yannuzzi LA, Slakter JS, Sorenson JA, Hanutsaha P, Spaide RF, et al. Classification of choroidal neovascularization by digital indocyanine green videoangiography. Ophthalmology.1996;103(12):2054-60.

3. Verteporfin Roundtable Participants. Guidelines for using verteporfin (Visudyne) in photodynamic therapy for choroidal neovascularization due to age-related macular degeneration and other causes: update. Retina. 2005;25(2):119-34.

4. Avery RL, Pieramici DJ, Rabena MD, Castellarin AA, Nasir MA, Giust MJ Intravitreal bevacizumab (Avastin) for neovascular age-related macular degeneration. Ophthalmology. 2006; 113(3):363-72.e5. Comment in: Ophthalmology. 2007;114(2):400; author reply 400-1.
5. Spaide RF, Laud K, Fine HF, Klancnik JM Jr, Meyerle CB, Yannuzzi LA, et al. Intravitreal bevacizumab treatment of choroidal neovascularization secondary to age-related macular degeneration. Retina. 2006;26(4):383-90.

6. Michels S, Rosenfeld PJ, Puliafito CA, Marcus EN, Venkatraman AS. Systemic bevacizumab (Avastin) therapy for neovascular age-related macular degeneration twelve-week results of an uncontrolled open-label clinical study. Ophthalmology. 2005; 112(6):1035-47.

7. Bom Aggio F, Eid Farah M, Melo GB. Intravitreal bevacizumab for occult choroidal neovascularization with pigment epithelium detachment in age-related macular degeneration. Acta Ophthalmol Scand. 2006;84(5):713-4

8. Laser photocoagulation of subfoveal neovascular lesions of age-related macular degeneration. Updated findings from two clinical trials. Macular Photocoagulation Study Group. Arch Ophthalmol. 1993;111(9):1200-9. Comment in: Arch Ophthalmol. 1994; 112(7):874-5.

9. Liew G, Mitchell P. Ranibizumab for neovascular age-related macular degeneration N Engl J Med. 2007;356(7):747-8; author reply 749-50. Comment on N Engl J Med. 2006;355(14):143244. N Engl J Med. 2006:355(14):1419-31.

10. Forooghian F, Cukras C, Meyerle CB, Chew EY, Wong WT. Tachyphylaxis after intravitreal bevacizumab for exudative age-related degeneration. Retina. 2009;29(6):723-31

\section{Simpósio Internacional Moacyr Álvaro - SIMASP}

\section{8 a 10 de março de 2012}

Maksoud Plaza Hotel São Paulo - SP

\section{Informações:}

Meeting Eventos

Tels.: (11) 3849-8263/3849-0379

Site: http://www.oftalmo.epm.br/simasp2012 